\title{
Streptococcal diseases of group B, its screening efficiency and role in neonatal and gynecological practice
}

\author{
Linda Luguze', Anna Abramova ${ }^{2 *}$ \\ ${ }^{1}$ Children's Clinical University Hospital, Riga, Latvia \\ ${ }^{2}$ Resident of Obstetrics and Gynaecology Program, Rigas Stradiņš University, Riga, Latvia
}

Received: 07 July 2015

Accepted: 22 July 2015

\section{*Correspondence:}

Dr. Anna Abramova,

E-mail: abramova.anna.4@gmail.com

Copyright: $\odot$ the author(s), publisher and licensee Medip Academy. This is an open-access article distributed under the terms of the Creative Commons Attribution Non-Commercial License, which permits unrestricted non-commercial use, distribution, and reproduction in any medium, provided the original work is properly cited.

\begin{abstract}
Beta-haemolytic streptococcus of group B (BGS) is one of the main reasons for perinatal morbidity and mortality in developed countries. It is one of the key initiators of early sepsis and meningitis in new-born period. BGS is the cause of neonatal death for 1 new-born per 10000 . Prospectively maternity histories and new-born development histories of the Riga Maternity House in the period from 01.07.2013 to 31.12.2013 were analyzed. All in all 712 randomly selected records were examined, children born from 37th to 42nd week of pregnancy (median 39th week of pregnancy), with a birth weight of 2100 to 4880 grams, of which 373 were girls and 339 boys. The study included both neonates, whose mothers during pregnancy were screened for streptococcus of group B, and those, whose mothers were not screened, or the result was not known at the time of birth. The data were processed and statistically analyzed using programs MS Excel and SPSS Statistics 20. Results of SPSS Statistics 20 were compared using the chi-squared test. Out of 712 pregnant women viewed, who should have been screened according to national procedure, it was done and the result at the time of birth was known for 502 pregnant women $(71 \%)$. Of 502 pregnant women who were screened, a positive result, which means that a woman was a BGS carrier, was confirmed in 87 cases $(17 \%)$. Intrauterine infection of new-born, whose mothers were screened positive, was found in 15 cases (17\%), and new-born, whose mothers were screened negative - in 24 cases or $6 \%$. The only sepsis risk factor found in a study group was anhydrous childbirth period that is longer than 18 hours. The number of carriers of Group B streptococcus in Latvia coincides with the average number in the world. Although the number of antenatally unobserved pregnant women in Latvia is only around 2\%, Group B streptococcus screening coverage of pregnant women is limited. Even when adequate prophylactic antibiotic therapy is received, it does not fully eliminate the possibility of intrauterine infection.
\end{abstract}

Keywords: Obstetric, Group B streptococcus, Neonatal, New-born, Screening, Pregnancy, Prophylactic, Sepsis, Perinatal mortality, Latvia, Riga

\section{INTRODUCTION}

\section{Characteristics of Group B streptococcus}

Group B beta-haemolytic streptococcus (BGS) or Streptococcus agalactiae is a facultative anaerobic grampositive coccus. In the blood agar feed coccus makes greyish-white 3-4 mm large colonies, around which there is a narrow beta haemolysis area. ${ }^{1}$ The BGS does not form spores and it has no capsules. At the room temperature it can live for several hours, can be killed if exposed to iodine, phenol, mercury dichloride, as well as a temperature greater than $60^{\circ} \mathrm{C} .^{2}$

While identifying coccus, it should be looked for the typical Lancefield carbohydrate antigen of group B. The surface of the bacteria is covered with polysaccharide 
capsule, by which the bacteria are divided into serotypes. At the moment following serotypes are displayed: IA, IB, II, III, IV, V, VI, VII, VIII. ${ }^{1,3}$ In US and Europe the most common types for people are IA, II, III and V. ${ }^{2}$

The bacteria's ability to cause invasive disease is determined by different virulence factors. One of them is the polysaccharide capsule and its structure, for invasive strains it is much more complicated. It has been proved that serotype III has expressed tropism to brain membranes; therefore it is the most common type in meningitis cases. Also polysaccharide capsule contains short side chains, at the ends of which are $\mathrm{N}$-acetylneuraminic acids that prevent activation of complement system, opsonisation and phagocytosis of bacteria is not happening. Bacteria-produced beta haemolysin and cytolysin play an important role in directly triggering early neonatal sepsis because it facilitates the move of bacteria from the alveoli into the blood stream. ${ }^{1,3}$

\section{Group B beta-haemolytic streptococcus and intrauterine infection risk}

The main risk factor for the development of early BGS infection is maternal colonization. ${ }^{4}$ Approximately 10$30 \%$ of pregnant women are carriers. In the Eastern Europe BGS colonization of mothers is found in 19,7\% $29,3 \%$ of cases, in the Western Europe in $11 \%-21 \%$ of cases, in Scandinavia in $24,3 \%-36 \%$ of cases, in the Southern Europe in 6,5\%-32\% of cases. ${ }^{5}$ During Latvian study it was found that $17 \%$ of pregnant women are carriers. $^{5}$

Usually pregnant women have asymptomatic BGS, but sometimes it can be life-threatening. Colonization can lead to miscarriage, premature delivery or premature shell bursts. ${ }^{6}$ During pregnancy the most common diseases are urinary tract infection, cystitis, pyelonephritis, horioamnionitis and endometritis, development of which may be a risk factor for a caesarean surgery. Infection of the mother in childbirth may include febrile bacteremia. Postpartum sepsis case lethality can reach up to $35 \%$. $^{6,7,8}$

BGS bacteriuria occurs in approximately $2-7 \%$ of pregnant women, which indicates a strong genital colonization. Vertical transmission risk during vaginal birth for a child, whose mother is a BGS carrier, is up to $70 \%$, of which $1-2 \%$ develop BGS induced infection. ${ }^{4}$ Children, whose mothers are BGS carriers, are more than 25 times more likely to develop early neonatal infection than those, whose mothers are not BGS carriers. ${ }^{4}$ Although the presence of other risk factors significantly increases the risk of infection to the new-born, such as premature amniotic membrane rupture or premature birth cases, the infection is found in 63 cases per 1000 newborn, yet the large number of new-born, for whom the only risk factor for the development of an early infection is BGS identified for mother, shows its importance - 4.3 cases per 1000 new-borns. ${ }^{9}$
In the United States since 1970 as the main risk factor of early BGS infection is recognized maternal genital or gastrointestinal tract colonization. Subsequent studies showed that using prophylactic intravenous antibiotics during labour can protect from early neonatal infection during the first week of life. In 1996, American College of Obstetricians and gynecologist- ACOG, Center for Disease Control and Prevention- CDC) and in 1997 the American Academy of Pediatrics- AAP issued recommendations, which stated the use of antibiotics during labour to mothers who have an increased risk of infection transmission. In 2002, the guidelines were revised and recommended universal prophylactic therapy, based on an overall BGS screening of all pregnant women carried out in 35 th- 37th gestational week. CDC statistics indicate that BGS general screening, followed by antibacterial prophylaxis in childbirth is more than $50 \%$ more efficient than a risk factor-based strategy. After the introduction of universal screening of pregnant women and antibacterial prophylaxis in childbirth, the number of early neonatal sepsis cases in the US has fallen from 1.7 / 1000 new-born in 1990 to $0.340 .37 / 1000$ new-born in $2008{ }^{4}$

Antibiotic prophylaxis during labour along with adequate post-natal care of the new-born has reduced early cases of disease by $80 \% .^{10}$

In Latvia perinatal mortality rate compared to other EU countries is still relatively high - 8.8 per 1,000 new-born in 2012. ${ }^{3}$ During Maternal and Child Health Improvement Plan since August of 2012 in Latvia as well was introduced BGS screening, which includes plating from the vagina, perineum and rectum for all pregnant women in 35th-37th pregnancy week. In case of positive screening mother receives prophylactic antibiotic therapy during labour. ${ }^{1}$ Screening aims to prevent or ease BGS related illness of new-born by reducing perinatal mortality and improving children's future quality of life. ${ }^{1}$

\section{Group B haemolytic streptococcus disease during neonatal period}

From BGS-related diseases the first is marked early neonatal sepsis. ${ }^{4}$

During new-born period, based on the age of manifestations of the disease, clinic and epidemiological data two main syndromes can be distinguished - early and late BGS disease.

About early BGS infection is spoken when the first symptoms of new-born appear up to 7 days of life, but about early neonatal sepsis- if clinical picture of sepsis manifested itself during the first 72 hours of life (3 days). ${ }^{8,11}$ In $80 \%$ of cases BGS caused illness shows during the first 24 hours. ${ }^{12}$ Early BGS infection is usually associated with pregnancy complications, such as urinary tract infections, horioamnionitis, premature amniotic membrane rupture or premature birth. If the mother is a 
carrier of BGS, a new-born can become infected with these microorganisms by going through colonized maternal genital. However, more severe cases are usually associated with ascending infection when the BGS gets into the amniotic fluid, which new-born aspire, causing respiratory tract colonization and possible further penetration of the micro-organism in the blood and spread to other organs. Early neonatal BGS infection mostly manifests itself as sepsis (50-80\% of cases), pneumonia $(30 \%)$ or meningitis $(5-15 \%)$, in rare cases asymptomatic bacteraemia. ${ }^{3,12,13}$ Generally BGS infection develops rapidly, leading to septic shock and generally very high mortality $(10-15 \%)^{3,12,14}$, which varies depending on the gestational age of the new-born- for premature new-born mortality from infectious BGS can reach $20-30 \%$, for term new-born- $2-3 \% .^{15}$

If the infection shows later- from the 7 th to 90 th day of life - it is regarded as late BGS disease, which is usually not related to the mother or birth complications, and up to the moment of infection no deviation from the norm of age of a new-born is observed. Bacteremia without focal infection focus is incident in $45-60 \%$ of late cases. The most serious late-BGS-specific expression of the disease is considered to be meningitis that develops in $25-35 \%$ of cases. Meningitis is characterized by sudden onset, rapid progression of the disease and seizures, for half of the patients leaving lasting neurological consequences. Lethality in late BGS disease cases is $2-6 \%$. Sometimes during late illness of new-born is observed arthritis, osteomyelitis or cellulitis. ${ }^{1,3,6}$

\section{Group B streptococcus infection clinic for new-born}

BGS infection symptoms are generally non-specific, so during the early diagnosis it is important to identify risk factors and discern early non-specific neonatal activity and dietary changes. ${ }^{16}$

Distress during labour may be the first sign of infection:

1. Foetal tachycardia during childbirth - shows the stress to be caused by intrauterine infection ${ }^{16}$;

2. Meconial amniotic fluid - indicates foetal distress and is associated with double risk for sepsis ${ }^{16}$;

3. Low Apgar scale score - indicates neonatal distress immediately after birth and is associated with early neonatal sepsis development. ${ }^{16}$ In Washington study for new-born with Apgar scale score under six, sepsis developed 36 times more often than for newborn with Apgar scale score over six. ${ }^{17}$

The most common symptoms of BGS infection are ${ }^{16}$ :

1. Temperature changes- increased, decreased or normal ${ }^{16}$ Usually fever for timely neonates appears in the second, the third day of life. ${ }^{18}$

2. Respiratory symptoms- tachypnea, wheezing, nasal wing raise, chest retraction, apnea;

3. Anorexia;
4. Cyanosis;
5. Jaundice;
6. Agitation;
7. Lethargy;
8. Decreased tissue perfusion;
9. Hypotension.

Sepsis symptoms range from nonspecific to severe septic shock. Both early sepsis BGS and BGS pneumonia may be associated with persistent pulmonary hypertension. ${ }^{18}$

Early BGS infectious meningitis rarely manifests with neurological symptoms. More often symptoms and respiratory symptoms and apnea. ${ }^{18}$

\section{Group $B$ streptococcus infection in laboratory diagnostics}

Blood plating- it is necessary for final confirmation of diagnosis. ${ }^{16,18}$ New-born weighing more than three kilograms must take at least one blood sample with a volume of three milliliters before antibiotic treatment. ${ }^{16}$

Full blood analysis is optimally done 6-12 hours after birth. ${ }^{16}$ In a multicenter study of new-born who were born after 34 weeks of gestation and whose sepsis developed during the first 24 hours, after taking blood tests and plating, it was shown that low total white blood cell count $(<5,000 /$ microL), absolute $(<1000 /$ microL) or relative $(<5000 /$ microL) neutropenia or immature polymorfnuclear and total polymorfnuclear white blood cell ratio that is greater than 0.3 , is associated with positive cultures and demonstrates BGS sepsis. ${ }^{18}$ Total white blood cell count of more than $20000 /$ microL, and the platelet count change are not indicators of early sepsis, but thrombocytopenia may indicate septic shock. ${ }^{19}$

C- reactive protein (CRP) is a non-specific indicator because its increase may be caused by non-infectious causes, such as fever of the mother, foetal distress, childbirth stress, perinatal asphyxia, meconium aspiration and intraventricular haemorrhage; it is not a reliable indicator for early diagnosis of sepsis. However, it can be used as an additional diagnostic criterion - if the CRP level does not increase in the dynamics, it is not unlikely that sepsis BGS will develop. If the CRP level drops lower than $0.1 \mathrm{mg} / \mathrm{dL}$ within 24-48 hours after initiation of treatment with antibiotics, probably a new-born is not infected and it is not needed to continue antibiotic therapy. ${ }^{16}$

Procalcitonine- in the precursor of calcitonin, which is released in response to bacterial toxins. Procalcitonine is considered to be a relatively reliable indicator the approval of bacterial infection in febrile neonates. ${ }^{16}$

Lumbar puncture is recommended in the following $\operatorname{cases}^{16}$ :

- Positive blood culture; 
- Clinic that indicates sepsis;

- Other laboratory data indicates sepsis;

- Clinical worsening at the background of adequate antibiotic therapy.

For new-born with sepsis clinic it is recommended to take plating from both blood and liquor, because blood culture can be a sterile in $15-33 \%$ of meningitis cases. ${ }^{4}$

Chest X-ray - should undergo new-born with respiratory symptoms. ${ }^{16} \mathrm{X}$-ray characteristics show pleural effusion, which helps to distinguish BGS pneumonia from hyaline membrane disease. ${ }^{18}$

\section{Group B streptococcal infections prevention in early neonatal period}

In 2010, the CDC guidelines included the recommendations for neonatologists to reduce unjustified laboratory tests and empirical use of antibiotics. ${ }^{4}$

There is no significant difference found in sepsis clinic between the new-born whose mothers received prophylactic therapy and those whose mothers did not, and in $90 \%$ of cases sepsis clinic developed within the first 24 hours. ${ }^{4}$ Preventive therapy during labor can affect the growth of BGS in plating of blood and liquor, but it does not affect the development of the clinic during the first 24-48 hours [20]. Leukocyte formula changes are affected and their diagnostic importance is only in 41$68 \%$ of cases. ${ }^{4,10}$

In AAP and CDC edited guidelines is prompted such action algorithm ${ }^{4,10}$

1. If a child has symptoms of sepsis, a full investigation must be performed and empirical antibiotic therapy initiated.

- Full diagnostics include: blood plating, complete blood analysis, including white blood cell and platelet counts, chest x-ray (if the new-born has respiratory symptoms), and lumbar puncture (if the situation is sufficiently stable to withstand the procedure).

- Antibiotic therapy should be directed against the most common pathogens of sepsis: Group B streptococcus, Escherichia coli and other gramnegative pathogens. Usually ampicillin or gentamicin is chosen

2. If there are no symptoms of sepsis, but there is a suspicion of horioamnionitis of mother, there must be an incomplete investigation plan completed and, if necessary, empirical antibiotic therapy initiated. Firstly, however, it is advisable to consult a gynaecologist and obstetrician about how reasonable is suspicion of horioamnionitis.

- Limited diagnostics include: blood plating, complete blood analysis, including white blood cell and platelet counts. It is recommended to perform blood tests at least 6 to 12 hours after birth.

- Empirical antibiotic therapy should be discontinued, as soon as the results of the analysis, excluding the diagnosis of sepsis, are known.

3. If the mother during pregnancy and childbirth did not have risk factors that determine the use of antibiotics during labour, the routine new-born care should be performed. However if signs of sepsis are found a full investigation must be performed and empirical antibiotic therapy initiated.

4. If the mother during labour has had some risk factors and received prophylactic treatment with intravenous penicillin, ampicillin or cefazolin at least four hours before the birth the routine new-born care and observation in hospital for at least 48 hours is performed. If a child has symptoms of sepsis, a full investigation must be performed and empirical antibiotic therapy initiated.

5. If the mother during childbirth has not received adequate antibiotic therapy, but the new-born was born after 37 weeks of gestation and dry period was shorter than 18 hours, the new-born is observed in hospital for at least 48 hours. If a child has symptoms of sepsis, a full investigation must be performed and empirical antibiotic therapy initiated.

6. If the mother during childbirth has not received adequate antibiotic therapy, but the new-born was born before 37 weeks of gestation and dry period was longer than 18 hours, then incomplete investigation plan must be performed and the newborn is observed in hospital for at least 48 hours.

\section{Therapy in the case of group B streptococcus infection}

If there is a suspicion of BGS sepsis, pneumonia, bacteremia or meningitis, it is recommended to initiate empiric therapy with ampicillin $(150 \mathrm{mg} / \mathrm{kg}$ every 12 hours, for meningitis every 8 hours) and gentamicin (4 $\mathrm{mg} / \mathrm{kg}$ once a day, for new-born born after 35 weeks gestation). ${ }^{18}$

If in the plating is confirmed BGS infection, treatment may be switched to monotherapy with penicillin $G$ (150,000 -200,000 U / kg per day, divided into three- four times, in case of meningitis 250,000 $-450,000 \mathrm{U} / \mathrm{kg}$ per day, divided into three times). ${ }^{18}$

The duration of treatment in case of sepsis, pneumonia and bacteremia is 10 days, but in case of meningitis 1421 day. $^{18}$

\section{BGS infection of new-born in Latvia in 2013}

The study included pregnant women aged 16-43 years with a median age of 29 years. 
New-born were divided into groups:

1. Mothers BGS screening was positive -87 new-born;

2. Mothers BGS screening was negative - 415 newborn;

3. Mothers screening was not carried out or at the time of birth the result was not known - 210 new-born.

In the selected groups was analyzed the number of newborn who developed neonatal infection. New born were compared within selected groups in order to assess whether screening result and applied preventive therapy affects the development of symptoms and course of the disease.

Assessing risk factors, in the case of which the pregnant woman during labour should receive prophylactic antibiotic therapy, none of the surveyed pregnant women were found fever during labour (> 380C) or BGS bacteriuria during pregnancy. Also anhydrous period longer than 18 hours is defined as a risk factor that increases the risk of infection for new-borns. ${ }^{4,9}$ The only risk factor found in the study group was anhydrous period in labour for more than 18 hours. However in the assessed study group anhydrous period longer than 18 hours was not found to be a risk factor, which significantly increases the risk of infection in new-born. In literature data of both study groups were included premature and full-term new-born, so it is likely that a lengthy anhydrous period more directly affects prematurely born. As this study deals only with full-term new-born, significant effects were not observed. Also, knowing that the anhydrous period longer than 18 hours is considered to be a risk factor, perhaps the obstetricians are trying to expedite birth faster for mothers with known positive BGS screening.

Intrauterine infection of new-born, whose mothers were screened positive, was found in 15 cases or $17 \%$. But for new-born, whose mothers were screened negative - in 24 cases or $6 \%$. Comparing the percentage of infected frequency it appeared that for BGS carriers neonatal infection occurs almost three times more often ( $p$ $<0.001)$. For new-born, whose mothers were not screened or at the time of birth results were not known, intrauterine infection was observed in 13 cases or $6 \%$. In comparison to new-born whose mothers were screened positive, in this case as well infection was found nearly three times more often and statistically derived significant difference $(\mathrm{p}=0.003$ ). In comparison to new born, whose mothers were screened negative, and mothers, who were not screened or at the time of birth results were not known, a statistically significant difference in terms of infection was not found $(\mathrm{p}=0.838)$. Generally in 35 childbirth cases anhydrous period was found to be longer than 18 hours.

For new-born in group 1 anhydrous period longer than 18 hours was in 2 cases or $2.3 \%$, while the for new-born in group 2 in 23 cases or 5,5\%, yet for those, whose mothers were not aware of the result of screening, in 10 cases or 4 $8 \%$. Comparing statistically the results by study groups, in none of the cases was obtained statistically significant difference.

Prophylactic antibiotic therapy was given to 110 mothers. In 85 cases $(77 \%)$ of the total 110 , which should have received prophylactic antibiotic treatment, it would not be used if the screening was not introduced. Other reasons why mothers received prophylactic antibiotic therapy were BGS + mothers with other risk factors for infection ( 2 cases) and BGS + mothers without any risk of infection (23 cases). New-born of Group 1, distress in childbirth found for one new-born or $6.7 \%$, but for neonates of Group 2 distress was found in two cases or $8.3 \%$; while comparing no statistical difference was obtained ( $\mathrm{p}=0.849$ ). For new-born of Group 3, distress found in 3 cases, or $23.1 \%$, but compared to new-born, whose mothers were screened positive of BGS, no statistical difference was obtained $(p=0.216)$. No statistical difference was obtained between new-born of Group 2 and 3 as well $(\mathrm{p}=0.210)$.

For none of the new-born in Group 1 Apgar scale score in the first minute less than six points was found. Compared to new-born in the Group 2, the score below six points was in six cases $(25 \%)$, which is statistically significantly more frequently $(\mathrm{p}=0.035)$. For the new-born in Group 2 Apgar scale score in the first minute less than six points was found in 4 cases (30.8\%) and in comparison to the new-born of Group 1 statistically significant difference was obtained $(p=0.02)$. Yet no statistically significant difference was obtained between new-born of Group 2 and 3 as well $(\mathrm{p}=0.706)$.

New-born of Group 1 in $40 \%$ of the cases were diagnosed in the first day, while new-born of Group 2 in $25 \%$ of cases. In cases where the mother was not aware of the result of screening, diagnosis in the first day was established in $46 \%$ of cases. However, a statistical difference between groups in diagnosis on the first day is not observed.

The average time for the initiation of antibiotic treatment for new-born of Group 1 was 1.6 days, while in the Group 2- 1.75 days. For new-born of Group 3 the average time to initiation of antibiotic treatment was 1.77 days. Comparing the average initiation time of antibiotic treatment between groups, no statistically significant difference was determined.

The average duration of therapy was 3.47 days for newborn of Group 1. The average duration of therapy for new-born of Group 2 was 3.88 days. Among these groups no statistical difference was found $(\mathrm{p}=0.119)$. The average duration of therapy for new-born of Group 3 was 4.62 days. Comparing the average duration of therapy between groups 2 and 3, no statistically significant difference was determined $(p=0.068)$. However, comparing the average duration of therapy between 
groups 1 and 3, a statistically significant difference was determined $(p=0.020)$. Yet the average duration of treatment given to new-born, whose mothers were screened positive of BGS, is relatively shorter. Consequently, one might think that introduced screening and preventive therapy received during childbirth affects both the development of the disease and draws close attention to new-born, resulting in faster diagnosis and initiation of therapy, which avoids severe sepsis cases.

Three new-born (20\%) of Group 1 were treated in the neonatal intensive care unit (NICU). In NICU also were treated three new-born (13\%) of Group 2 and the difference between these groups is not statistically significant $(\mathrm{p}=0.52)$. NICU treated seven new-born (54\%) from the Group 3. Comparing Groups 1 and 3, as well as 2 and 3 were obtained statistically significant differences $(\mathrm{p}=0.05$ and $\mathrm{p}=0.007)$.

\section{CONCLUSION}

Although the number of antenatally unobserved pregnant women in Latvia is only around $2 \%$ Group B streptococcus screening coverage of pregnant women is limited. Group B streptococcus screening introduced in Latvia has contributed to the early diagnosis of neonatal infection, while positive screening results that lead to antibacterial therapy have reduced the incidence of severe Group B streptococcal disease cases. New-borns', whose mothers' Group B streptococcus screening was positive and who received prophylactic antibiotic therapy during labour, symptoms immediately after birth, indicating early development of infection, were observed less frequently. In cases where the mother during pregnancy is diagnosed with Group B streptococcus, for new-born in case of infection antibiotic treatment started earlier and the time of treatment is shorter.

\section{Funding: No funding sources \\ Conflict of interest: None declared \\ Ethical approval: Not required}

\section{REFERENCES}

1. Sarah S. Long, MD, Charles G. Prober, MD and Larry K. Pickering, MD, "Principles and Practice of Pediatric Infectious Diseases", 4th Edition, Chapter 119: Streptococcus agalactiae (Group B Streptococcus)2012.708-713.

2. National Institute for Health and Clinical Excellence, Antibiotics for early-onset neonatal infection", NICE clinical guideline 149, 2012. Available at: http://publications.nice.org.uk/antibiotics-for-earlyonset-neonatal-infection-cg149 Accessed 16 Mart, 2014.

3. Robert M. Kliegman, MD, Bonita M.D. Stanton, MD, Joseph St. Geme, Nina Schor, MD, PhD and Richard E. Behrman, MD. "Nelson Textbook of Pediatrics", 19th Edition, Chapter 177 - Group B Streptococcus, 925-928.
4. Jennifer R. Verani, MD, Lesley McGee, PhD Stephanie J. Schrag, Dphil "Prevention of Perinatal Group B Streptococcal Disease Revised Guidelines from CDC, 2010" Morbidity and Mortality Weekly Report Recommendations and Reports, November 19, 2010.59/10.1-32.

5. Barcaite E, Bartusevicius A, Tameliene R. u.c. "Prevalence of maternal group B streptococcal colonization in European countries." Department of Obstetrics and Gynaecology, Kaunas University of Medicine, Lithuania, 2008. Available at: http://www.ncbi.nlm.nih.gov/pubmed/18307064 Accessed 18 April,2014.

6. M. J. Soto Marió, I Valenzuela, u.c. "Prevention of Early-onset Neonatal Group B Streptococcal Disease" Available at: http://www.ncbi.nlm.nih.gov/pmc/articles/PMC3861 949/\#!po=12.5000 Accessed 1 April 2014.

7. Ann L Anderson-Berry, MD; Chief Editor: Ted Rosencrantz "Neonatal sepsis" 2012. Available at: http://emedicine.medscape.com/article/978352overview\#aw2aab6b2b3 Accessed 19 April 2014.

8. Tami H. Skoff, Monica M. Farley, Susan Petit "Increasing Burden of Invasive Group B Streptococcal Disease in Nonpregnant Adults, 19902007', Clinical Infectious Diseases 2009. 49/1, 8592.

9. Sagori Mukhopadhyay, Karen M. Puopolo „Risk Assessment in Neonatal Early-Onset Sepsis" [tiešsaiste], 2012 December. Available at: http://www.ncbi.nlm.nih.gov/pmc/articles/PMC3782 302/ Accessed 1 May 2014.

10. Committee on Infectious Diseases and Committee on Fetus and Newborn "Recommendations for the Prevention of Perinatal Group B Streptococcal (GBS) Disease", PEDIATRICS, Official journal of the American Academy of Pediatrics, August 1, 2011,128/3,611-616.

11. National Institute for Health and Clinical Excellence, "Antibiotics for early-onset neonatal infection", NICE clinical guideline 149,2012. Available at: http://publications.nice.org.uk/antibiotics-for-earlyonset-neonatal-infection-cg149 Accessed 1 May 2014.

12. Anne Schuchat "Epidemiology of Group B Streptococcal Disease in the United States: Shifting Paradigms" $1998 . \quad$ Available at: http://www.ncbi.nlm.nih.gov/pmc/articles/PMC8889 3/\#!po=5.43478 Accessed 7 July 2014.

13. Sarah S. Long, MD, Charles G. Prober, MD and Larry K. Pickering, MD „Principles and Practice of Pediatric Infectious Diseases", 4th Edition, Chapter 119: Streptococcus agalactiae (Group B Streptococcus) 708-713.

14. Tami H. Skoff, Monica M. Farley, Susan Petit. Increasing Burden of Invasive Group B Streptococcal Disease in Nonpregnant Adults, 19902007. Clinical Infectious Diseases. 2009;49(1):8592. 
15. The Society of Obstetricians and Gynaecologists of Canada (SOGC) "The Prevention of Early-Onset Neonatal Group B Streptococcal Disease" 2013;298:1-10.

16. Morven S Edwards, MD „Clinical features and diagnosis of sepsis in term and late preterm infants" 2014. Available at: http://www.uptodate.com/contents/clinical-featuresand-diagnosis-of-sepsis-in-term-and-late-preterminfants. Accessed 3 May 2014.

17. Soman M, Green B, Daling J. "Risk factors for early neonatal sepsis", $1985 . \quad$ Available at: http://www.ncbi.nlm.nih.gov/pubmed?term=401416 3 Accessed 9 June 2014.

18. Karen M Puopolo, MD, PhD Carol J Baker, MD "Group B streptococcal infection in neonates and young infants" 2014. Available at: http://www.uptodate.com/contents/group-bstreptococcal-infection-in-neonates-and-younginfants. Accessed 15 June 2014.

19. Karen M Puopolo, MD, PhD Carol J Baker, MD "Management of the infant whose mother has received group B streptococcal chemoprophylaxis" $2014 . \quad$ Available at: http://www.uptodate.com/contents/management-ofthe-infant-whose-mother-has-received-group-bstreptococcal-chemoprophylaxis. Accessed 10 July 2014.

Cite this article as: Luguze L, Abramova A. Streptococcal diseases of group B, its screening efficiency and role in neonatal and gynaecological practice. Int J Reprod Contracept Obstet Gynecol 2015;4:934-40. 